\title{
EXPERIMENTAL MODEL
}

\section{New combinatorial optogenetic mouse lines for cardiovascular biology}

Lee, F.K. et al. elife 10, e67858 (2021)

Optogenetics is a technique that uses light to dynamically perturb the function of light-sensitive protein effectors (often rhodopsin-containing molecules) that are genetically expressed in the cells or tissues of interest. Optogenetic effectors, combined with cell lineage specific promoters, enable the experimental manipulation of discrete cell populations through optical activation of membrane channels or secondary messengers. Genetically encoded optical sensors are a different type of optogenetic tools that can be used to monitor cell activity: optogenetic sensors include genetically-engineered fluorescent calcium indicators (GECIs) that increase their fluorescence upon binding to $\mathrm{Ca} 2+$, a key signaling ion involved in different cell functions, including heart and blood vessels contraction. By allowing precise spatiotemporal control and monitoring over intracellular signalling processes in vivo, optogenetic tools can provide valuable insights into biological processes.

In a new study, Frank K. Lee and colleagues report the development of the CHROMus (The Cornell/National Heart
Lung Blood Resource for Optogenetic Mouse Signaling), a new mammalian optogenetic toolbox for cardiovascular research. The toolbox consists of multiple, optically-compatible effector/sensor optogenetic mouse lines designed for combinatorial experiments within the cardiovascular system. Specifically, they generated 21 lines of optogenetic sensor and effector mice, in which the transgenes were selectively expressed in cardiac pacemaker cells, cardiomyocytes, endothelial cells, smooth muscle cells, alveolar epithelial cell, lymphocytes and glial cells mainly, under cell-specific promoters. The optogenetic effectors selected were the rhodopsin/ adrenergic receptor fusion proteins optoo1AR, optoß2AR, and protein CatCh2. Light activation of these molecules in the different cardiac cells resulted in increased levels of inositol trisphosphate receptor (InsP3) and cAMP secondary messenger pathways, and activation of $\mathrm{Ca} 2+$ membrane channel, respectively. The fluorescent GECI proteins, GCaMP and RCaMP were derived from circularly permutated EGFP and RFP constructs for in vivo, real-time reporting of cellular $\mathrm{Ca} 2+$ signalling in the different cell types. The researchers demonstrated the lineage-specific expression and optical function of the different optogenetic effectors and sensors, and tested some of the new optogenetic mouse lines for combinatorial experiments. In the biallelic sinoatrial (SA) nodal CatCh2/ cardiomyocyte-GCaMP8 mouse, activation of the CatCh2 protein expressed in SA nodal pacemaker cells triggered cardiac conduction, as monitored by GCaMP8 fluorescence in ventricular myocytes.

The derivation of these new optogenetic sensor/effector mouse lines will help to elucidate clinically-relevant, physiological and pathological molecular and cellular interactions in the cardiovascular system. Monoallelic lines can further be crossed with mouse models of cardiovascular diseases to investigate disease pathophysiology.

\section{Anna Melidoni}

Published online: 23 December 2021 https://doi.org/10.1038/s41684-021-00906-9

\section{natureresearch} ACADEMIES

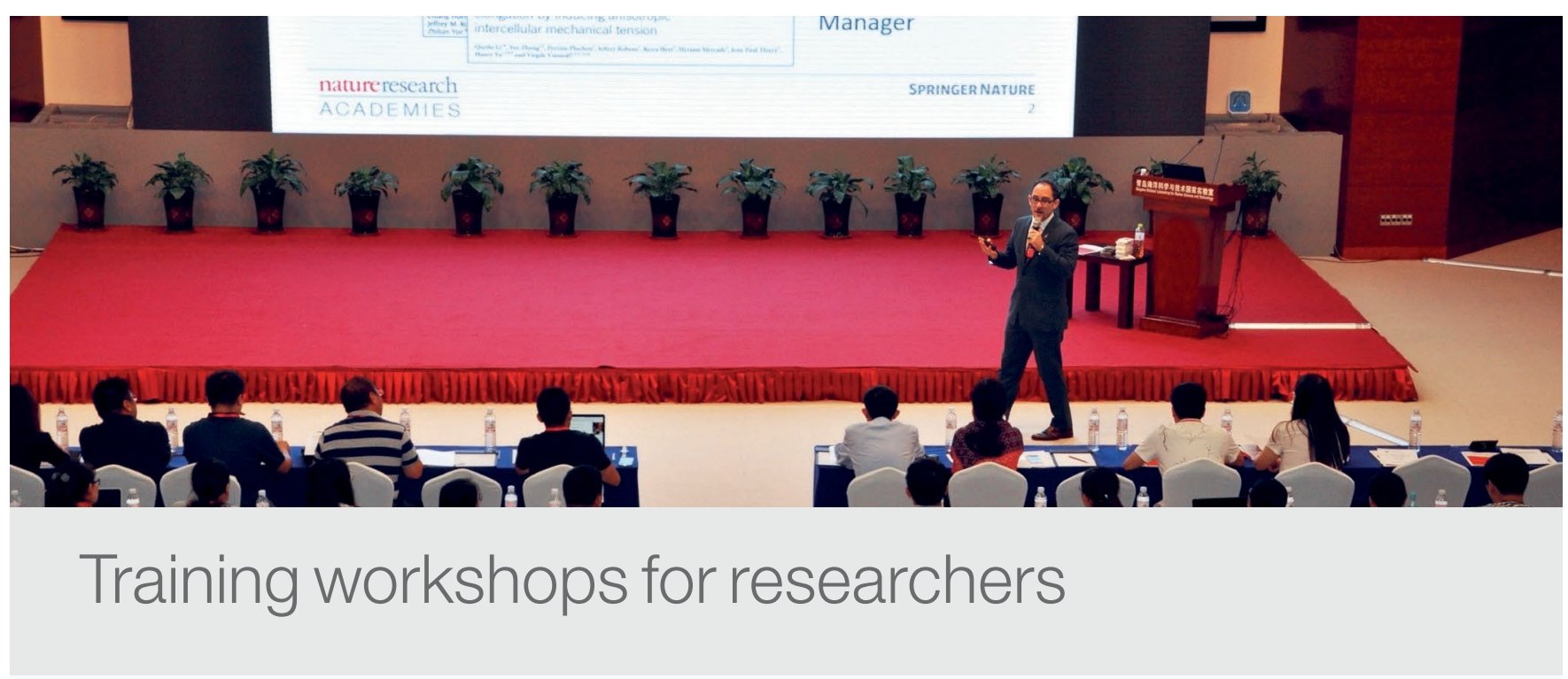

A series of workshops to support researchers, covering topics such as getting published, journal editing, clinical research methodology, and applying for research positions.

Visit partnerships.nature.com/academies to host an academy at your institution. 